\title{
Diversity As Technology: A New Perspective
}

Francis Daniel, Belmont University, USA

\begin{abstract}
The business case for diversity is really a technological one. Like the Hubble telescope uses multiple lenses to acquire an understanding of the universe that no one lens can achieve alone, the business case suggests that diversity (however defined) provides multiple perspectives that help organizations better understand an increasingly complex and heterogeneous marketplace. If the prevailing argument for diversity is indeed technology-driven, the present paper suggests that looking at diversity through the lens of the Technology Acceptance Model 3 (TAM3) might provide insights that could improve both the acceptance of diversity in the organization and its impact on organizational performance.
\end{abstract}

Keywords: Workplace Diversity; Technology Acceptance Model 3 (TAM3); Business Case for Diversity

\section{INTRODUCTION}

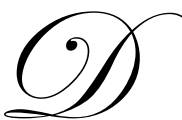

iversity management has grown into an $\$ 8$ billion dollar industry centered on making diversity more palatable and useful to organizations not accustomed to difference (Hansen, 2003). These organizations face the prospect of increased diversity for a myriad of reasons from changing demographics, increased government and societal pressure, to the need to better understand and find competitive advantage in an increasingly diverse marketplace. It is this last reason, commonly referred to as the "business case for diversity", that most top managers and diversity proponents use as the main selling point for accepting/welcoming/seeking diversity within organizations. It has been suggested that diverse teams and organizations outperform their homogeneous counterparts, with increased creativity and perspective providing a sustainable competitive advantage that can drive performance (Swan, Polzer, Seyle, \& Ko, 2004). Evidence for the diversity-performance relationship in the literature has been inconclusive and often anecdotal (Prieto, Phipps, \& Osiri, 2009). The clear line between the $\$ 8$ billion dollar investment and the promised return has been difficult to draw, and business has been seeking guidance in how to achieve the acceptance and appropriate deployment of diversity that leads to improved performance and bottom line results. Theory and practice in diversity management has to date, been unable to provide that guidance.

At its core, the business case involves viewing diversity as a form of technology. Much like the Hubble Space telescope, whose multiple lenses allow it to see along a broader spectrum of light than can the human eye, diverse companies gain multiple simultaneous views of a single issue or competitive landscape that when put together can provide a clearer and more accurate picture than their homogeneous competitors can achieve. But like all technology, diversity has not been universally effective or accepted in the settings it was intended to serve.

The present paper will investigate diversity "technology" (its acceptance and deployment) using the Technology Acceptance Model 3 (TAM3) framework. This approach offers a new way of looking at the diversity issue and the challenges associated with developing effective diversity management strategies and programs. TAM3 identifies aspects of technology acceptance that need to be addressed in the diversity management context such as diversity's perceived usefulness and ease of use, and whether adoption is voluntary or mandated. These factors, among others have been shown to affect the ultimate acceptability of new technology in the workplace. Viewing diversity technology through the TAM3 lens might provide insights that could improve both the acceptance of diversity in the organization and its impact on organizational performance, as well as anticipate the probability of acceptance and success for future diversity initiatives. 


\section{TECHNOLOGY ACCEPTANCE MODELS}

Technology acceptance is a mature stream in the Information Systems (IS) field that has sought to identify the factors that lead individuals in organizations to try and ultimately adopt new information technologies (IT) in the workplace. The importance of this question is obvious when one considers that each failure of a new IT deployment to 'take' in a large organization can result in the loss of hundreds of millions of dollars (Koch, 2004; Venkatesh \& Bala, 2008). Numerous theories and models have been proposed over the years to explain technology acceptance, the most prominent of which is Davis' (1989) Technology Acceptance Model (TAM). The basic premise of TAM is that an individual's intention to try a new technology (behavior intent) and the actual use thereof (use behavior) are positively related to that individual's perception of how useful the technology is (Perceived Usefulness (PU)) and how easy it is to use (Perceived Ease of Use (PEU)) (see figure 1). PU is formally defined as "the degree to which a person believes that using a particular system would enhance his or her job performance", while PEU is "the degree to which a person believes that using an IT will be free of effort (Davis, 1989, p. 320). Simple yet robust explaining roughly $40 \%$ of an individual's intent and actual use of IT, TAM has been tested many times on a variety of technologies and continues to garner strong empirical support in the IS literature (Venkatesh \& Bala, 2008). TAM has also been used successfully to study other adoption decisions outside of the traditional IS realm in areas such as marketing (Dabholkar \& Bagozzi, 2002; Gentry \& Calantone, 2002; Yang \& Peterson, 2004), advertising (Roger \& Chen, 2002), and green electricity use (Arkesteijn \& Oerlemans, 2005). The predicted relationship also proved valid in international studies in cultures as varied as the UK (Foster, 2005), Japan (Straub, 1994; Straub, Keil, \& Brenner, 1997), China (Vankatesh \& Zhang, 2010), and Saudi Arabia (Abdulgader \& Kozar, 1995).

While TAM is by far the most studied and used technology acceptance model with over 1000 citations of the two seminal articles (Davis, 1989; Davis et al. 1989) that introduced TAM (Venkatesh, Davis, \& Morris, 2007; Venkatesh \& Bala, 2008), it is not without its limitations. TAM has evolved over time in response to criticisms about its simplicity and failure to elaborate on what factors actually lead to usefulness and ease of use determinations. Critics argued that without this fundamental understanding, it would be impossible to design the types of programs and interventions needed to maximize the likelihood of deployment success. TAM researchers stepped in to fill this conceptual void, identifying four general types of determinants of perceived usefulness and ease of use - individual difference (personality and demographic traits), system characteristics (salient features of the IT system under consideration that can lead to positive or negative perceptions of PU and PEU), social influence (social processes and mechanism that help form opinion/perceptions), and facilitating conditions (organizational support that facilitates IT use) (Venkatesh, 2000; Venkatesh \& Davis, 2000) . Venkatesh and Bala (2008) incorporated and elaborated on, all of these earlier findings resulting in the Technology Acceptance Model 3 (TAM3) that will inform the discussion that follows.

\section{Technology Acceptance Model 3}

Determinants of PU. In TAM3 (Venkatesh \& Bala, 2008), the determinants of PU include perceived ease of use, subjective norm, image, job relevance, output quality and result demonstrability (See figure 1). As in many other areas of life, an individual's perception of IT can be colored by social influences separate from the objective characteristics of the technology being considered. In TAM 3, these social influences are represented by subjective norm and image. Subjective norm is defined as "the degree to which an individual perceives that most people who are important to him think he should or should not use the proposed IT" and image is "the degree to which an individual perceives that use of an innovation will enhance his or her status in a social system" (Venkatesh \& Bala, 2008).

The other four determinants of PU are system characteristics, referred to by the authors as cognitive instrumental processes (Venkatesh \& Bala, 2008), which focus on the characteristics of the IT and its potential to positively impact work processes and outcomes. Job relevance is the degree to which an individual believes that the target system is applicable to his or her job. Output quality is "the degree to which an individual believes that the system performs his or her job tasks well". Result demonstrability reflects the degree to which an individual believes that the results of using the IT are tangible, observable, and communicable (Moore \& Benbasat, 1991). Perceived ease of use is the only determinant in this category whose effect on perceived usefulness is moderated by experience. 




Study results suggest that perceived ease of use, subjective norm, image, and result demonstrability are all significant and consistent predictors of PU. Job relevance and output quality were found to have an interactive effect on PU, with the effect of job relevance on PU increasing with increases in output quality. Also important to note is the finding that experience moderated the relationship between subjective norm and PU, with subjective norm's impact diminishing as one gains experience with the new technology (Venkatesh, 2000; Venkatesh \& Bala, 2008).

Determinants of PEU. The determinants of PEU in TAM 3 were developed using the anchoring and adjustment framing of human decision making that suggests that individuals will form early perceptions of ease of use based on anchors related to individuals' general beliefs regarding the technology of interest and its use. These early perceptions are then adjusted after hands-on experience with the new technology (Vankatesh, 2000; Venkatesh \& Bala, 2008). Venkatesh (2000) suggests four anchors associated with PEU, three of which reflect individual differences (computer self-efficacy, computer anxiety, and computer playfulness), the last a facilitating condition (perception of external control). The system characteristics-related adjustments are in the form of perceived enjoyment and objective usability.

Computer self-efficacy is "the degree to which an individual believes that he or she has the ability to perform a specific task/job using a computer". Computer anxiety is the degree of "an individual's apprehension, or even fear, when she/he is faced with the possibility of using computers", while computer playfulness is "the degree of cognitive spontaneity in microcomputer interactions (Webster \& Martocchio, 1992, p. 204). Perception of external control is "the degree to which an individual believes that organizational and technical resources exist to 
support the use of the new system (Vankatesh et al., 2003). Perceived enjoyment is the degree to which using the new system or technology is perceived to be enjoyable in its own right, aside from any performance consequences resulting from its use (Venkatesh, 2000) and finally, objective usability is a comparison of the new system versus other systems based on the actual (rather than perceived) level of effort required to complete specific task (Venkatesh, 2000).

Consistent with TAM 3 expectations, results indicate that the anchors - computer self-efficacy, perception of external control, computer anxiety, and computer playfulness are consistent predictors of PEOU. On the other hand, the effect of the adjustments - perceived enjoyment and objective usability- on PEOU are shown to grow as experience with the technology increases. Conversely, the effect of computer anxiety on PEOU diminishes with increasing experience (Venkatesh, 2000; Venkatesh \& Bala, 2008)

\section{Behavioral Intention and Use}

Tests of the TAM3 framework find that perceived usefulness is the strongest predictor of behavioral intent. The effect of perceived ease of use on behavioral intent is also significant at the beginning of the new technology deployment but gradually diminishes as experience with the new technology increases and reality displaces perception. A three-way interaction among subjective norm, experience, and voluntariness ("the degree of free will involved in the adoption of an information system" (Wu \& Lederer, 2009)) to which adoption of new technology is perceived to be voluntary versus mandatory) on behavioral intent is also supported, such that the effect of subjective norm on behavioral intent weakens with increased experience with the new technology, particularly in a voluntary context. In a two-way interaction between subjective norm and voluntariness, the effect of subjective norm on behavioral intent is stronger in a mandatory context. Finally, and also consistent with prior findings, behavioral intent is a significant predictor of use in TAM3.

\section{Other Relevant Technology Adoption Findings}

There are several other findings from twenty years of technology adoption research that are particularly relevant to the discussion of diversity from a technology perspective that follows. First, while TAM 3 and other models suggest possible determinants of perceived usefulness and perceived ease of use, the literature is consistent in its contention that the appropriate mix and effect of determinants is dependent on the technology being introduced. Therefore, each new technology and each setting need to be viewed as a unique circumstance that will require researchers and practitioners, alike to thoroughly investigate how characteristics of the new technology interact with characteristics of the setting to impact the relationship between perceived usefulness, perceived ease of use, and their determinants (Venkatesh \& Bala, 2008). Second, PU and PEU do not crystallize at implementation, but continue to be formed over time and experience with the technology. This means that the positive perceptions and intentions held at the time of deployment, where many companies concentrate their technology adoption efforts, may falter post deployment if not properly nurtured. Indeed, post-adoptive behavior models suggest that if use of new technology is not reinforced, the user will revert back to habitual behaviors and attitudes ( Jasperson, Carter, \& Zmud, 2005).

Particularly germane to the current discussion, both gender and age have been shown to have a moderating effect on the relationship between and among PU, PEU, and the various determinants of both. Specifically, women were found to be influenced more significantly by subjective norms than men in the determination of behavioral intent, while men tend to be influenced most by perceived usefulness (Venkatesh \& Morris, 2000; Yang, Hsu, \& Tan, 2010). Differences have been found in other demographic categories, as well. Porter and Donthu (2006) looked at race and economic status, along with age in using TAM to explore the impact of these differences on intent to use the internet and actual usage. Their findings suggest that each of these demographics have a significant impact on PU, PEU, intent, as well as actual usage of the internet.

\section{DIVERSITY THROUGH A TAM 3 LENS}

If one accepts the premise that diversity, as touted in the oft-used business case for diversity, represents a form of technology that top management can use as a source for competitive advantage, then obvious parallels can 
be drawn between the challenges both IS and diversity managers face in attaining organization-wide acceptance and usage of their respective systems. For instance, the business case for diversity is clearly a usefulness argument. Research has suggested that diverse teams outperform homogeneous teams because their multiple perspectives lead to greater creativity in solving complex or novel problems (Prieto et al., 2009). Likewise, organizations operating in a diverse marketplace gain advantage if their workforce reflects that diversity. They are able to understand and better serve their customers, attract and keep the best employees from a diverse labor pool. All of these diversityderived benefits lead to greater performance and higher profits.

As many an IS manager has learned, however, these benefits only accrue if the technology is adopted by, and used appropriately in the organization. As a technology, diversity has often been a difficult sale for 'ease of use'. In this regard, diversity management programs have often been their own worst enemies. Most of these programs concentrate on areas that have little to do with the technology aspect of diversity, working instead to improve the organization's socio-cultural climate. The same research that has shown promise for the diversity technology has also found problems. While it is true that diverse groups can outperform homogeneous ones, they can also be much more difficult to manage, have a harder time reaching consensus, and face higher dissatisfaction and turnover in membership. Diversity at the organizational level has often failed to deliver, as well. Part of this can possibly be attributed to poor deployment, where the technology does not reach some of the places in the organization where it is most needed (top management, board, i.e.). The consequences of a failed diversity deployment are the same as those discussed above for IS; lost money and time, and severely frayed emotions. As it has done so often for the IS industry, the TAM 3 framework (See figure 2) should offer insights into how to improve the PU and PEU determinations that lead to successful adoption and use of workplace diversity.

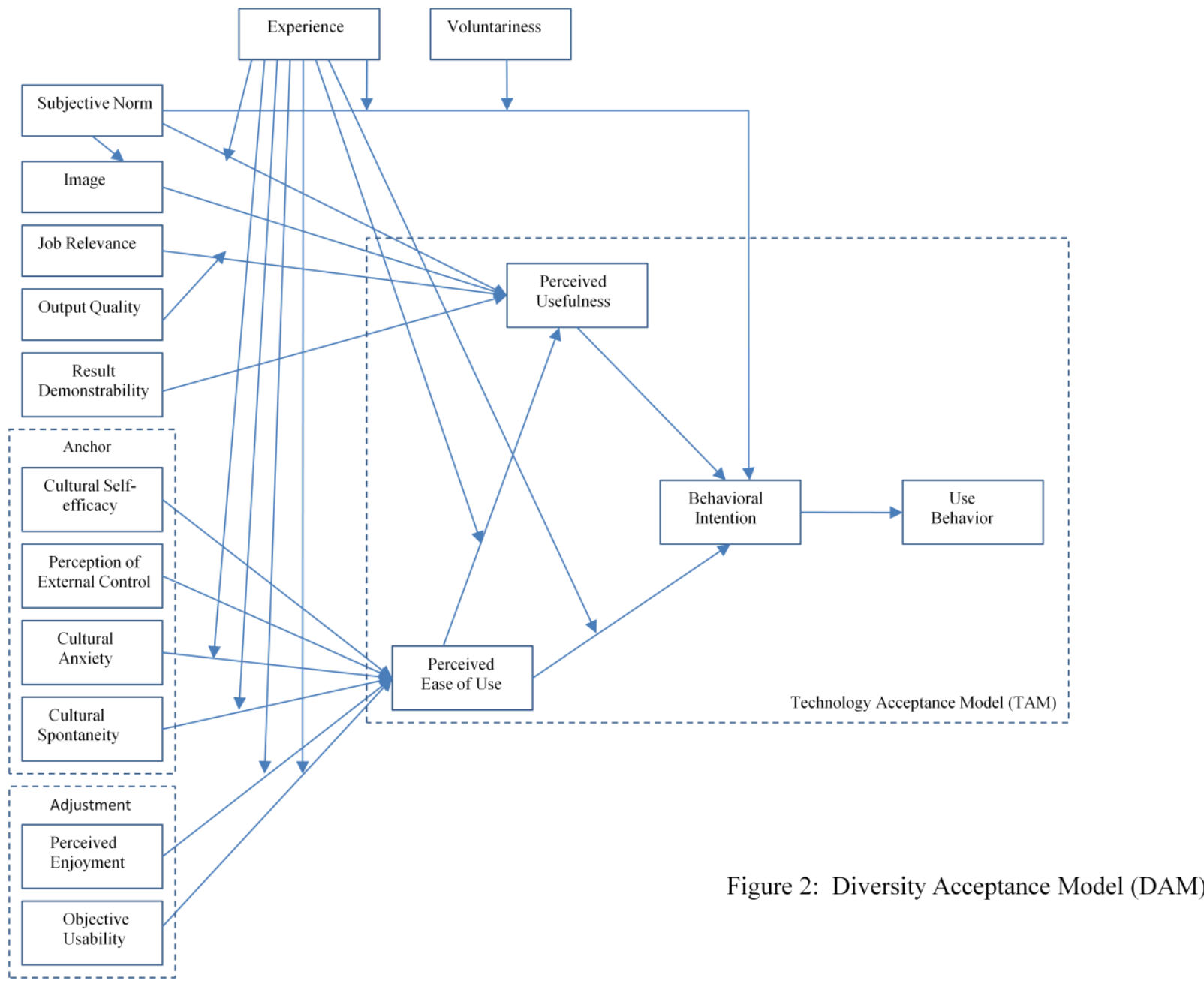




\section{Diversity's Perceived Usefulness}

System Characteristics. For all the trumpeting of the bottom-line benefits of diversity, very few companies actually quantify the return on their diversity investment (Hansen, 2003; Kochan et al., 2003). This may be because outside of turnover numbers or changing employee demographics, it can be difficult to pinpoint the technology's impact. Result demonstrability at both the macro and micro levels is challenging in this setting, and this indeed has been the biggest complaint from the critics of the business case for diversity. From a conceptual perspective, TAM 3 sees this as a problem, as well given result demonstrability's role in influencing perceived usefulness. Identifying and highlighting measurable outcomes is critical to result demonstrability, but is something that most companies are unable or unwilling to do (Hansen, 2003; Kochan et al., 2003)). Integrating the diversity technology into the work experience is important for several reasons, as well. Employees must be able to understand how the technology helps them do their job (Job relevance) and interact with it on a regular basis. Without this reinforcement, the technology will be abandoned and the workers will, as noted earlier revert back to their habitual behavior and attitudes - a decidedly negative outcome for a diversity initiative. Some of the most successful diversity programs operating today concentrate their efforts on teaching teams how to overcome the challenges associated with diverse teams and effectively use diversity technology to improve the quality of their decisions and other outcomes (Ely \& Thomas, 2001). Output quality moderates the effect of job relevance on PU. The more success with the technology, the more reinforcing this determinant can be.

Social Influences. Without the hard numbers to offer as concrete results, many businesses seem to be attacking PU through social influence. Consultants do cultural audits and lead interventions designed to change the value system of the organization as it relates to diversity. If successful at getting everybody onboard, this approach should have a positive impact on subjective norm (assuming your colleagues at work are among those whose approval you seek). It also makes sense that a change in organizational culture and values would redefine symbols of status (image), as well. However, TAM3 does offer a warning for companies counting on social influence to drive diversity technology use (and profits). Subjective norm's direct effect on PU is moderated and weakened over time by experience. If the company fails to address the other PU determinants before subjective norm's effect diminishes $\mathrm{PU}$, the most impactful force driving behavioral intention and use behavior, will simply run out of fuel.

\section{Diversity's Perceived Ease of Use}

Anchors. In applying TAM 3 to diversity, the conceptualization (and terminology) associated the individual differences require the most (though still subtle) adjustment. In the same way that general experience with and affect towards computers underpins initial perceptions of any and all IS technology, general experience with and affect towards culture (yours and others') should inform initial perceptions about diversity technology. Computer self-efficacy becomes cultural self-efficacy, the degree to which an individual believes that he or she has the ability to perform a specific task/job in a culture rich environment, something akin to a self-assessment of one's cultural IQ (Earley, 2003; Earley \& Ang, 2003).. Similarly, cultural anxiety is the degree of an individual's apprehension, or even fear, when she/he is faced with the possibility of having to deal with a culture rich environment. Every good and (especially) bad experience one has had that could be attributed to someone else' 'difference' comes into play, here. Computer playfulness becomes cultural spontaneity, and still seeks to capture the wonder of the unexpected interactions that happen in cultural exchanges. Businesses seem to get this part very right or very wrong in the run-up to deployment. The literature abounds with stories of consultants who initiate horribly divisive interventions (damaging efficacy and spontaneity, raising anxiety levels), then leave before resolving the volatile situation they created (perception of external control). These anchors set the tone, the initial perception for everything that follows. Interventions should build confidence and good will, relieving the anxiety and providing assurance that the organization is committed for the long haul. .

Adjustment. If the anchors were about anticipation, the adjustments are about realization. From management's perspective, you want the reality of working with the diversity technology to be as impactful and rewarding as possible. Perceived enjoyment and objective usability speak to the ongoing nature of the assessment process wherein perceptions are constantly modified by experience and acted upon. Management has to remain committed, both with resources and attention, for this deployment to take hold in the organization. For technology 
to be perceived as usable, it must actually be used. This is critical to the learning process that must inform the further development and evolution of the technology and supporting infrastructure.

\section{Using Diversity Technology}

The same can be said for perceptions of usefulness and ease of use that these and the other constructs help to determine. Much of diversity management intervention appears focused on creating a welcoming and fair environment where all people can feel that they matter and have an opportunity for success - or at least would not be openly ostracized. This is a noble and necessary goal and clearly reflects one very important aspect of the diversity management construct. But, TAM seems to suggest that for diversity technology to have a chance to deliver on the business promises top management made for it and through it, the technology has to be tied to specific tasks, and then help perform those tasks better than the individuals could without it. Diversity as a technology has to perform, and perform consistently well to guarantee its long-term viability. It should be noted that the systemfocused antecedents (job relevance, output quality, result demonstrability) are the only ones whose effect on PU is not directly or indirectly moderated by experience or diminished over time. The other part of that equation is that the tasks have to matter, to dwell at a level where improved perception and vision can influence decisions that direct the entire organization. The technology has to be usable and useful and properly used. No one of these alone can guarantee long-term use.

\section{FUTURE DIRECTION}

The diversity management field has been around for over two decades, and by many accounts has failed to find its footing, its identity. The purpose of this exercise was to re-imagine, re-conceptualize one part of the diversity management paradigm - the business case for diversity - so that it could be looked at in a new way, through a different lens. Re-framing diversity as technology may ultimately fail to gain much traction among diversity management researchers, but exposing that technology to TAM scrutiny has raised some interesting avenues for future investigation.

First, not every aspect of this analogy is applicable to every challenge faced in trying to manage diversity. There are many reasons why diversity increases in an organization and many arguments for why that increase should be welcomed - and why certain types of diversity should not. The present paper has focused solely on the business case aspect. It remains to be seen whether the Technology Acceptance approach could provide support to the acceptance and implementation of diversity management based solely on the socio-cultural argument (Valuing diversity is the right thing to do). There are some competitive advantages to be gained through valuing employee differences (reduced turnover, greater satisfaction, less stress, greater motivation, etc.), but these gains are not the main thrust of this argument.

Technology acceptance literature has its own diversity stream, albeit not as well developed as our own. Their studies have looked at the impact of gender, age, and race diversity on PU, PEU, behavioral intent and other constructs when dealing with technology adoption. How do these relationships change when the 'technology' is the diversity, itself? In other words, would diversity effects be different for social versus technical interventions?

\section{CONCLUSION}

The present paper investigated the adoption of diversity using the Technology Acceptance Model 3 (TAM3) frameworks. This approach offers a new way of looking at the diversity issue and the challenges associated with developing an effective diversity strategy and program(s). TAM 3 identified aspects of technology acceptance that need to be addressed in the diversity management context such as diversity's perceived usefulness and ease of use, whether or not adoption is voluntary. All of these factors have been shown to affect the ultimate acceptability of new technology in the workplace. The diversity as technology perspective also provides more options to researchers and practitioners, alike. Diversity as technology is neither right nor wrong, so managing diversity becomes a strategic issue rather than a moral imperative. Finally, this framework could also be used as a predictive model to anticipate the probability of success for future diversity initiatives. 


\section{AUTHOR INFORMATION}

Francis Daniel, Ph.D., is an Assistant Professor of Management at Belmont University in Nashville, Tennessee. His research focuses on slack resources, trust, and diversity management issues. He has published in the Journal of Business Research, Industrial Management and Data Systems, and Decision Sciences Journal of Innovative Education.

\section{REFERENCES}

1. Abdul-Gader, A.H. \& Kozar, K.A. (1995). The Impact of Computer Alienation on Information Technology Investment Decisions: An Exploratory Cross-national Analysis. MIS Quarterly, 19(4),. 535-559.

2. Arkesteijn, K. \& Oerlemans, L. (2005). The Early Adoption of Green Power by Dutch Households: An Empirical Exploration of Factors Influencing the Early Adoption of Green Electricity for Domestic Purposes. Energy Policy, 33(2), 183-196.

3. Dabholkar, P.A. \& Bagozzi, R.P. (2002). An Attitudinal Model of Technology-Based Self-Service: Moderating Effects of Consumer Traits and Situational Factors. Journal of the Academy of Marketing Science, 30(3), 184-201.

4. Davis, F.D. (1989). Perceived Usefulness, Perceived Ease of Use, and User Acceptance of Information Technology. MIS Quarterly, 13(3), 319-340.

5. Davis, F.D., Bagozzi, R.P., \& Warshaw, P.R. (1989). User Acceptance of Computer Technology: A Comparison of Two Theoretical Models. Management Science, 35(8), 982-1003.

6. Earley, P. C. (2003). Redefining interactions across cultures and organizations: Moving forward with cultural intelligence. Research in Organizational Behavior, 24, 271-299.

7. Earley, P. C. \& Ang, S. (2003). Cultural intelligence: An analysis of individual interactions across cultures. Palo Alto, CA: Stanford University Press.

8. Ely, R. J. \& Thomas, D. A. (2001). Cultural Diversity at Work: The Effects of Diversity Perspectives on Work Group Processes and Outcomes. Administrative Science Quarterly, 46 (2), 229-273.

9. Foster, C. (2005). Implementing Diversity Management in Retailing: Exploring the Role of Organizational Context. International Review of Retail, Distribution and Consumer Research, 15 (4), 471-487.

10. Gentry, L. \& Calantone, R. (2002). A Comparison of Three Models to Explain Shop-Bot Use on the Web. Psychology and Marketing, 19(11), 945-956.

11. Hansen, F. (2003). Diversity's Business Case Doesn't Add Up. Workforce, April, 28-32.

12. Jasperson, J., Carter, P.E., \& Zmud, R.W. (2005). A Comprehensive Conceptualization of Post-Adoptive Behaviors Associated With Information Technology Enabled Work Systems. MIS Quarterly, 29 (3), 525557.

13. Kochan, T., Bezrukova, K., Ely, R., Jackson, S., Joshi, A., Jehn, K., Leonard, J., Levine, D., \& Thomas, D. (2003). The Effects of Diversity on Business Performance: Report of the Diversity Research Network. Human Resource Management, 42 (1), 3-21.

14. Moore, G. C. \& Benbasat, I. (1991). Development of an instrument to measure the perceptions of adopting an information technology innovation. Information Systems Research, 2, 192-222.

15. Porter, E.P. \& Donthu, N. (2006). Using the Technology Acceptance Model to Explain How Attitudes Determine Internet Usage: The Role of Perceived Access Barriers and Demographics. Journal of Business Research, 59, 999-1007.

16. Prieto, L.C., Phipps, S.T.A., \& Osiri, J.K. (2009). Linking Workplace Diversity to Organizational Performance: a Conceptual Framework. Journal of Diversity Management, 4(4), 13-21.

17. Rodgers, S. \& Chen, Q.M. (2002). Post-Adoption Attitudes to Advertising on the Internet. Journal of Advertising Research, 42(5), 95-104.

18. Straub, D.W. (1994). The Effect of Culture on It Diffusion - E-Mail and Fax in Japan and the UnitedStates. Information Systems Research, 5(1), 23-47.

19. Straub, D.W., Keil, M., \& Brenner, W. (1997). Testing the Technology Acceptance Model Across Cultures: A Three Country Study. Information and Management, 33(1), 1-11.

20. Swan, W. B., Polzer, J. T., Seyle, D. C., \& Ko, S. J. (2004). Finding Value in Diversity: Verification of Personal and Social Self-Views in Diverse Groups. Academy of Management Review, 29(1), 9-27. 
21. Venkatesh, V. (1999). Creation of Favorable User Perceptions: Exploring the Role of Intrinsic Motivation. MIS Quarterly, 23(2), 239-260.

22. Venkatesh, V. \& Bala, H. (2008). Technology Acceptance Model 3 and a Research Agenda on Interventions. Decision Sciences, 39(2), 272-315.

23. Venkatesh, V. \& Davis, F.D. (2000). A Theoretical Extension of the Technology Acceptance Model: Four Longitudinal Field Studies. Management Science, 46(2), 186-204.

24. Venkatesh, V. \& Davis, F.D. (1996). A Model of the Antecedents of Perceived Ease of Use: Development and Test. Decision Sciences, 27(3), 451-481.

25. Venkatesh, V. \& Morris, M.G. (2000). Why Don't Men Ever Stop to Ask for Directions? Gender, Social Influence, and Their Role in Technology Acceptance and Usage Behavior. MIS Quarterly, 24(1), 115-139.

26. Venkatesh, V., Morris, M.G., \& Ackerman, P.L. (2000). A Longitudinal Field Investigation of Gender Differences in Individual Technology Adoption Decision-Making Processes. Organizational Behavior and Human Decision Processes, 83(1), 33-60.

27. Venkatesh, V., Morris, M.G., Davis, G.B., \& Davis, F.D. (2003). User Acceptance of Information Technology: Toward a Unified View. MIS Quarterly, 27(3), 425-478.

28. Venkatesh, V., \& Speier, C. (1999). Computer Technology Training in the Workplace: A Longitudinal Investigation of the Effect of Mood. Organizational Behavior and Human Decision Processes, 79(1), 1-28.

29. Venkatesh, V. \& Zhang, X. (2010). Unified Theory of Acceptance and Use of Technology: U.S. Vs. China. Journal of Global Information Technology Management, 13(1), 5-27.

30. Webster, J., \& Martocchio, J. J. (1992). Microcomputer playfulness: Development of a measure with workplace implications. MIS Quarterly, 16, 201-226.

31. Wu, J. \& Lederer, A. (2009). A Meta-Analysis of the Role of Environment-Based Voluntariness in Information Technology Acceptance. MIS Quarterly, 33 (2), 419-432.

32. Yang, Z.L., \& Peterson, R.T. (2004). Customer Perceived Value, Satisfaction, and Loyalty: The Role of Switching Costs. Psychology and Marketing, 21(10), 799-822.

33. Yang, C., Hsu, Y., \& Tan, S. (2010). Predicting the Determinants of Users' Intentions for Using YouTube to Share Video: Moderating Gender Effects. Cyberpsychology, Behavior, and Social Networking, 13 (2), $141-152$. 


\section{NOTES}

